\title{
Profissionais de saúde e sua relação com a morte e o morrer de pacientes em UTI
}

\author{
Health professionals and their relationship with the death and dying of patients in ICU \\ Profesionales de la salud y su relación con la muerte y la muerte de pacientes en UCI \\ Mayra Rodrigues Leite ${ }^{1 \star}$, Nádia Maria Silva Montelo.
}

\begin{abstract}
RESUMO
Objetivo: Verificar a relação dos profissionais de saúde de um Centro de Terapia Intensiva frente à morte e o morrer dos pacientes. Métodos: Trata-se de estudo exploratório, descritivo com uso de método qualitativo. Participaram 11 profissionais de saúde de cinco categorias profissionais, atuantes em unidades de terapia intensiva adulta e pediátrica de um hospital público da Amazônia Ocidental. Os dados foram coletados por meio de entrevista semi-estruturada e submetidos à análise de conteúdo proposta por Bardin. Resultados: Foram levantadas três categorias de análise. Nelas, constataram-se sentimentos de impotência, indiferença e tristeza, além de significados como fim de sofrimento e de causa transcendental. Tais sentimentos e significados podem coexistir e são atravessados por fatores característicos do paciente, do profissional e da relação entre ambos. Os profissionais reconhecem a necessidade de suporte psicológico, de conscientização sobre o processo de morte e aplicação dos princípios de cuidados paliativos. Conclusão: Visto que a convivência com a morte pode afetar negativamente esses sujeitos, principalmente quanto à competência profissional, verifica-se a necessidade de ações voltadas à educação para a morte como meio de instrumentalização desses profissionais.
\end{abstract}

Palavras-chave: Morte, Profissionais de Saúde, Unidade de Terapia Intensiva.

\begin{abstract}
Objective: To verify the relation of health professionals from an Intensive Care Center when facing the death and dying of patients. Methodologies: This is an exploratory and descriptive study using the qualitative method. 11 health professionals from five different professional categories participated, acting in adult and pediatric intensive care units from a public hospital from Western Amazon. The data was collected through semi-structured interview and submitted for content analysis as proposed by Bardin. Results: three categories of analysis were raised. In them, feelings of impotence, indifference and sadness were found, as well as meanings as an end to suffering and a transcendental cause. Such feelings and meanings can coexist and are crossed by characteristic factors from the patient, the professional and also from the relation between both. Professionals recognize the necessity of psychological support, awareness about the process of death and application of the principles of palliative care. Conclusion: Since living with death can negatively affect these subjects, specially with regard to professional competence, there is a need for actions aimed at education for death as a means of instrumentalizing these professionals.
\end{abstract}

Keywords: Death, Health professionals, Intensive Care Unit.

\section{RESUMEN}

Objetivo: Verificar la relación de los profesionales de la salud de un Centro de Terapia Intensiva ante la muerte y el óbito de los pacientes. Métodos: Se trata de un estudio exploratorio, descriptivo con método cualitativo. Tuvieron la participación de once profesionales de la salud de cinco categorías que trabajan en Unidades de Cuidados Intensivos para adultos y pediátricos de un hospital público de la Amazonía Occidental. Los datos fueron colectados a través de entrevistas semiestructuradas y sometidos al análisis de contenido propuesto por Bardin. Resultados: Fueron levantadas tres categorías de análisis. En ellos se encontraron sentimientos de impotencia, indiferencia y tristeza, además de que significados como fin del sufrimiento y causa trascendental. Tales sentimientos y significados pueden coexistir y son atravesados por

\footnotetext{
${ }^{1}$ Hospital Regional de Cacoal (HRC), Cacoal - RO. * E-mail: mayrarleite123@gmail.com
} 
factores característicos del paciente, del profesional y da relación entre ellos. Los profesionales reconocen la necesidad de apoyo psicológico, de concientización sobre el proceso de muerte y aplicación de los principios de los cuidados paliativos. Conclusión: En razón de que la convivencia con la muerte puede afectar negativamente a estos sujetos, principalmente en lo que respecta a la competencia profesional es necesario realizar acciones orientadas a la educación para la muerte como medio de instrumentalización de estos profesionales.

Palabras-clave: Muerte, Profesionales de la salud, Unidad de Cuidados Intensivos.

\section{INTRODUÇÃO}

As Unidades de Terapia Intensiva são locais organizados e preparados com aparato tecnológico e recursos humanos especializados para onde são direcionados pacientes graves. O hospital, de maneira geral, é considerado um lugar cujo objetivo é o tratamento de enfermidades, a recuperação da saúde com foco na manutenção da vida. Porém nem sempre é dessa forma, havendo momentos nos quais a morte acontece.

A maneira de vivenciar a morte sofreu modificações no decorrer do tempo, sendo diferente em cada cultura. O processo de morte não esteve sempre associado ao hospital. Quando alguém adoecia, os cuidados eram comumente prestados por familiares nos lares. Todavia, com o avanço da medicina, os doentes passaram a estar nas mãos de profissionais. Desse modo, o que era percebido com mais naturalidade e familiaridade, passou a ser visto com maior distanciamento, espanto e evitação pela sociedade (ARIES P, 2017).

Os sentimentos provindos de situações de morte abarcam não apenas familiares e o paciente, mas também os profissionais da saúde (KOVACS MJ, 2010). Vicensi (2016), ao elaborar um estudo bibliográfico no qual reflete sobre a morte e o morrer na perspectiva de profissionais da UTI, mostra que eles se veem diante de um paradoxo. Ao mesmo tempo em que a UTI é local de pacientes recuperáveis, é também de pacientes terminais. Apesar de muitos profissionais buscarem não ter envolvimento com o paciente (PEREIRA NETO HS, et al., 2019) para evitar que seu contexto de trabalho interfira na vida pessoal, isso não é completamente possível.

Monteiro JK (2012) realizou um estudo com enfermeiros e destacou a morte dos pacientes como um dos aspectos do trabalho em UTI que contribui para o sofrimento psíquico do profissional. Abordou ainda que o convívio ser habitual não impede que os profissionais sofram diante dessa realidade, que chegam a viver um dilema ético entre ser técnico e ser humano. Outro estudo evidenciou que o convívio com angústias de pacientes e familiares, considerando as perdas por morte, provoca desgaste nos profissionais, sendo inclusive motivo de absenteísmo (ABREU RMD, et al, 2014).

No contexto ocidental, a morte é considerada um tabu, o que a torna um tema pouco ou dificilmente abordado. Além disso, dentro dos hospitais, angústias frente à morte de pacientes costumam não serem reconhecidas e autorizadas (KOVACS MJ, 2010), havendo um cenário que não colabora com uma vivência saudável por parte dos profissionais. Isso pode desencadear prejuízos na saúde da equipe e, consequentemente, do paciente, devido às suas características que demandam emocionalmente dos profissionais (ALBUQUERDE SGE, 2015).

Desta maneira, considerando as repercussões que essa situação pode acarretar na saúde mental e física do profissional e na assistência aos pacientes, o objetivo deste estudo foi verificar a relação dos profissionais de saúde de um Centro de Terapia Intensiva de um hospital da Amazônia Ocidental frente à morte e o morrer dos pacientes.

\section{MÉTODOS}

Trata-se de um estudo exploratório descritivo com uso do método qualitativo. Os dados foram coletados por meio de entrevistas semi-estruturadas com um roteiro composto por seis questões norteadoras e um questionário com dados sociodemográficos e profissionais. 
A instituição na qual os participantes atuam é um hospital público responsável pela segunda macrorregião do estado de Rondônia. No momento da pesquisa havia um total de 24 leitos de UTI, sendo duas atendendo público adulto e uma pediátrica com 10, oito e seis leitos, respectivamente.

A amostragem foi aleatória, composta por 11 profissionais da saúde de diferentes categorias (Enfermagem, Fisioterapia, Medicina, Psicologia, Serviço Social e Técnico de Enfermagem) atuantes há pelo menos três meses em alguma das unidades e que tivessem participado da primeira etapa. Isso porque serão apresentados aqui resultados parciais de uma pesquisa com método misto cujo primeiro momento consistiu na aplicação de uma escala, no qual participaram 72 profissionais pertencentes às profissões mencionadas.

Buscou-se inicialmente a participação de dois profissionais por categoria, abrangendo um de cada tipo de UTI. Por atuarem em ambos os tipos, Psicologia e Serviço Social tiveram apenas um de cada profissão. Devido a mudanças estruturais no hospital durante a coleta de dados, a unidade pediátrica foi desativada, sendo dificultado o contato com o médico desta unidade. Quanto aos profissionais a mais de outras profissões, ambos buscaram por conta própria participarem desta etapa, sendo incluídos pelas pesquisadoras.

As entrevistas aconteceram em junho de 2020. Foram posteriormente transcritas, codificadas conforme os temas frequentes e interpretadas com base na Análise de Conteúdo de Bardin. Ressalta-se que neste estudo serão transcritas parte das falas dos participantes.

A pesquisa iniciou após aprovação do Comitê de Ética e Pesquisa da Faculdade de Ciências Biomédicas com parecer número 3.718.254 e da Direção do hospital. Os participantes receberam e assinaram Termo de Consentimento Livre e Esclarecido.

\section{RESULTADOS}

\section{Caracterização da amostra}

Os participantes foram majoritariamente mulheres (72,73\%) com idade entre 21 e 55 anos, com média de 35,55 anos. A maioria era casada $(63,64 \%)$, seguida de pessoas solteiras $(27,27 \%)$ e as demais $(9,09 \%)$ divorciadas ou viúvas.

A amostra foi composta por seis enfermeiros e fisioterapeutas, sendo três de cada, seguido de dois técnicos de enfermagem, e um médico, um psicólogo e um assistente social. Dos participantes, seis atuavam na UTI adulto, três na pediátrica e dois em ambos os tipos de unidade.

Quanto ao tempo de atuação em UTI, cinco estavam há menos de dois anos, quatro entre dois e 10 anos, e dois há mais de 10 anos, sendo que alguns passaram por diversos setores hospitalares durante sua carreira profissional.

Por fim, os participantes atuavam no hospital de um a cinco dias por semana. Tal variação se deu pelas especificidades do vínculo de cada profissional com a instituição, sendo sete deles efetivos e os demais pós-graduandos residentes. Todos seguiam regime de plantão, alguns de 12 e outros de 24 horas.

Após a análise dos dados foram construídas três categorias, sendo elas: fatores determinantes no sofrimento do profissional; sentimentos e significados frente à morte dos pacientes; e possibilidades de intervenção sob a ótica dos profissionais.

\section{Fatores determinantes no sofrimento do profissional perante a morte do paciente}

No decorrer das falas, foi apontada maior sensibilidade e sofrimento dos profissionais quando diante da morte de crianças quando comparado a de adultos e idosos.

"[...] tenho uma sensibilidade maior quando se trata da pediatria. Mas na UTI [...] depende muito do caso, se é um paciente idoso, sem prognóstico, cheio de comorbidades, não me gera angustias. Mas se é um paciente novo, que teria a vida toda pela frente, com prognóstico positivo, isso mexe comigo sim" (E9 Psicologia). 
Todavia, quando o paciente apresenta prognóstico reservado, observa-se melhor aceitação por parte dos profissionais, mesmo ele sendo pediátrico.

"Aqui na UTI pediátrica também, as mortes que a gente teve foram mais de crianças com alguma má formação congênita, já tinha um histórico de outras patologias. Então você vai se conformando" (E1 Enfermagem).

Além disso, os participantes relataram constantes preocupações sobre seu desempenho profissional questionando-se se havia ou não sido adequado no cuidado ao paciente a fim de não causar-lhe dano. Conforme avaliassem sua conduta como acertada, sentiam-se mais tranquilos.

"[...] quando eu entrei na UTI a primeira vez, o que me gerou muito medo foi eu ser a causadora da morte do paciente, não dele morrer. Eu não tinha preocupação nenhuma se o paciente fosse morrer. Eu tinha preocupação se eu arrancasse algum dos fios que estava nele, se eu matasse ele" (E5 Fisioterapia).

"Mas o que me deixa mais tranquilo é que eu penso que eu fiz o que eu tinha que fazer" (E7 Fisioterapia).

O tempo de atuação em UTI e, consequentemente, a maior convivência com a morte de pacientes, foram apresentados como determinantes na qualidade de suas reações, sendo menor o impacto da morte dos pacientes conforme mais tempo de experiência.

"No inicio me afetava mais, agora já não me afeta tanto [...] Acho que com os anos isso vai se reduzindo. A gente encara a morte com um pouco mais de naturalidade" (E1 Enfermagem).

"Acho que como é tão rotineiro para a gente na UTI ter o óbito, acho que não importa mais [...], que acabou ficando fria para mim essa questão"

(E3 Enfermagem).

$\mathrm{Na}$ análise observou-se que as reações dos profissionais, isto é, os sentimentos e significados atribuídos a essas vivências de morte e morrer de pacientes, sofriam forte influência de fatores como os descritos. Dessa maneira, as reações apresentadas a seguir precisam ser observadas sob essa ótica.

\section{Sentimentos e significados frente à morte $\mathrm{e} o$ morrer dos pacientes}

Tendo em vista fatores anteriormente citados que atravessam os sentimentos vivenciados e significados atribuídos ante a morte e o morrer do paciente, alguns profissionais relataram não se sentirem afetados com essa vivência no trabalho.

"Eu digo isso porque o paciente que vem a óbito não mexe comigo" (E5 Fisioterapia).

"Com a morte, não. Nunca me incomodou e não me incomoda até hoje. Mas é mais fácil para mim porque eu me acostumei, não é algo que me abala emocionalmente" (E12 Técnico de Enfermagem).

Entretanto, o sentimento de impotência foi apresentado por alguns participantes como sendo frequentemente experienciado diante dessa realidade.

"Em algumas eu me sinto incapaz de realizar algo. É... Impotente. De querer realizar algo e não poder" (E1 Enfermagem).

Muito embora seja buscada uma relação estritamente profissional com o paciente, foi evidenciado nos profissionais destas unidades intensivas sentimentos como tristeza e abalo.

"Eu me sinto triste, muito mexida, abalada. Não dá para falar que é mais um, que eu não conhecia, porque a gente acaba pegando esse zelo esse carinho por eles [...]. Então, quando a gente lida com isso e chega até a hora de a gente participar da péssima notícia para a família a gente sente, [...] é muito desgastante, muito doloroso" (E2 Serviço Social). 
Sendo a UTI caracterizada por possuir recursos tecnológicos especializados para suporte de vida, muitas vezes, invasivos e que existem pacientes adoecidos e com internação de longa permanência, alguns profissionais atribuíram à morte do paciente o significado de descanso e fim do sofrimento.

"[...] a gente meio que espera ele descansar e às vezes a gente acha que isso é um certo alívio para o paciente, um descanso. Ele sofre muito na UTI. Então a gente acaba lidando com isso de uma forma melhor, como um descanso mesmo. Às vezes o paciente sofre muito com os procedimentos, a gente vê que não tem mais jeito" (E4 Enfermagem).

A UTI é um lugar de incertezas. Assim, embora haja recursos humanos e tecnológicos especializados para manterem a vida do doente, existem momentos que o desfecho é a morte. Quando o profissional reconhece tais esforços e verifica que apesar deles a morte acontece, atribui a esta um sentido transcendental.

"[...] é uma coisa que eu tento transferir como sendo uma coisa maior porque a gente vê que tem pacientes que tá tão fácil de a gente reverter [casos de parada cardíaca], mas você não vai reverter. [...] E outro caso, não tava voltando, fiz a atropina, que é feita após a parada, mas aí jogamos a toalha... Vamos parar. [...] E o paciente voltou (E8 Medicina)"

Depende do plano que Deus tem na vida de cada um. [...] Se ele determina que a pessoa virá para ficar um mês, aí com um mês a pessoa vai para ficar em outro plano" (E2 Serviço Social).

Além disso, tendo em vista a frequência de situação de morte, alguns profissionais compreendem ser esse evento parte do trabalho deles, algo que sabem que pode vir a acontecer e, nisso, não produz neles impacto tão significativo.

"Significa nada. Para mim não. É meu trabalho. Eu venho faço tudo o que eu posso. E não significa nada "ah perdi um paciente e fiquei triste". Não, não fiquei triste" (E12 Técnico de Enfermagem).

\section{Possibilidades de intervenções pela ótica dos profissionais}

Os profissionais entrevistados trouxeram sugestões de ações que poderiam ser aplicadas e, assim, facilitar a vivência de situações de morte e morrer de pacientes. Dentre elas, foi apontada a importância de haver suporte psicológico e educação para a morte.

"[...] acolhimento, uma conversa. Um momento - pode ser com todo mundo junto de conversa mesmo com psicólogo. Tentar entender o que é essa morte, o processo de morte" (E6 Fisioterapeuta).

"Acho que tinha que ter toda uma conscientização mesmo sobre o processo de morte" (E5 Fisioterapia).

Além disso, os participantes apontaram que a aplicação efetiva dos princípios de cuidados paliativos proporcionaria uma melhor relação dos profissionais com a morte dos pacientes.

"Acho que se tivessem cuidados paliativos efetivos eu conseguiria lidar melhor até na minha assistência, porque eu não evitaria vê-lo desconfortável porque se eu visse ele desconfortável eu ia conversar com o médico para ver o que a gente poderia fazer para dar um conforto. [...] Agora hoje, você ver um desconforto, estar lá, não ter nenhum tipo de protocolo, é como se eu tivesse ajudando a matar ele. [...] me sinto extremamente impotente. [...] eu sei que não tem como lutar contra a morte, eles vão morrer, mas eu me sinto impotente [...] de não ajudar ele a fazer a transição da melhor forma possível, ou da menos ruim. Porque é possível"

(E5 Fisioterapia). 


\section{DISCUSSÃO}

Conforme os resultados apresentados, existem elementos que influenciam a reação do profissional frente à morte do paciente. Dentre estes, a tendência ao maior sofrimento diante da morte de pacientes pediátricos comparado a de adultos ou idosos corrobora com outros estudos (BARBOSA AMGC e MASSARONI L, 2016, PERBONI JS, et al., 2018). A morte destes é avaliada como mais suportável, pois seguem uma cronologia esperada, sem haver uma interrupção do ciclo de vida (GONÇALVES JR e SIMOES JRS, 2019), diferente de quando acontece com crianças ou mesmo jovens.

Um estudo com profissionais de UTI pediátrica (NAVAIS MC, et al., 2017), aborda que neste ambiente é mais difícil não desenvolver vínculo afetivo ao cuidar dos pacientes, pois todo o contexto familiar está mais envolvido quando eles adoecem. Nisso, sentimento de empatia surge nos profissionais ao compartilharem o sofrimento dos familiares (SOUZA PSN e CONCEIÇÃO AOF, 2018).

Outro elemento presente foi a forma diferenciada da morte esperada e inesperada afetarem a intensidade do abalo emocional do profissional (SCARTON J, et al., 2013) sendo que, quando é súbita tende a causar mais impacto. Isso foi também identificado nos profissionais de UTI pediátrica que relataram um choque menor na morte de crianças com doenças crônicas ou prognóstico ruim (VIERAS AR e PIO DAM, 2018).

Caram CS, et al. (2018) discorrem que a morte muitas vezes é vista como um teste de competência profissional. Segundo eles, os profissionais tendem a sentirem-se tranquilos se avaliarem que fizeram o que estava ao seu alcance, mesmo o desfecho sendo a morte do paciente. Quando, porém, acontece o contrário - se seu desempenho for avaliado como inadequado - tendem a sentirem culpa ou afeto semelhante (PERBONI JS, et al., 2018), semelhantemente ao resultado identificado nesta pesquisa.

Outros estudos também demonstraram que profissionais atuantes há mais tempo em UTI tendem a se sentir menos afetados frente à morte de pacientes (BARBOSA AMGC e MASSORANI L, 2016). Isso se dá porque, mediante 0 contato constante com essa realidade, os profissionais desenvolvem estratégias para manejá-la, passando a estar supostamente mais bem preparados (GONÇALVES JR e SIMOES JRS, 2019).

O sofrimento tende a ser maior no acompanhamento e enfrentamento da morte de pacientes que ficaram internados por mais tempo e que puderam conhecer sua história de vida (SANTOS QN, et al., 2020). Maior permanência do paciente na unidade intensiva possibilita a construção de um vínculo com o profissional. $O$ rompimento desse vínculo produz sofrimento mesmo que a morte seja esperada, uma vez que é natural passar pelo luto quando diante de uma perda.

A convivência frequente desses profissionais com situações de morte de pacientes acaba provocando maior naturalização deste acontecimento, podendo desenvolver um enrijecimento tornando-o menos sensível diante da morte ali (BASTOS RA, et al., 2018). Tal reação é reforçada por atitudes como o distanciamento comum do profissional da saúde com o paciente (LIMA AMM, et al., 2019) tendo objetivo de evitar a construção de vínculo e o pesar, caso seja rompido. Assim, a naturalização é utilizada como estratégia para minimizar o sofrimento no cuidado desses pacientes (MOTA MS, et al., 2011)

Ademais, Santos QN, et al. (2020) ressaltam que a naturalização em si da morte nesse contexto não é um problema. O risco se dá quando, no lugar da dela, surge a mecanização da prática desenvolvida, pois assim os pacientes seriam objetos a serem manipulados e não pessoas a serem cuidadas.

Em outros casos, diferentemente, tal convivência tende a ocasionar uma série de sentimentos característicos desse momento, como culpa, angústia, tristeza, abalo, impotência, frustração (KOVACS MJ, 2010; HAYASIDA NMA, et al., 2014; MARTINS LA, et al., 2019), corroborando com o que foi relatado por alguns dos participantes desta pesquisa. Ressalta-se que, mesmo o profissional sabendo que o processo de morte faz parte do trabalho, o sentimento de impotência pode afetá-lo (MOTA MS, et al., 2011). Tais sentimentos são esperados, uma vez que os profissionais sentem-se responsáveis pela preservação da vida do paciente (GONÇALVES JR e SIMOES JRS, 2019).

Atribuir à morte do paciente o sentido de descanso é semelhante ao resultado obtido por Martins LA, et al. (2019) que afirmam que tal percepção é um modo de vivenciar situações de morte com menor angustia e 
sofrimento. Outro estudo, dessa vez com profissionais de UTI pediátrica, (VIEIRAS AR e PIO DAM, 2018) apresenta que a compreensão dos profissionais sobre a morte dos pacientes pode ter caráter de alívio devido o contexto de sofrimento que o paciente e a família vivenciavam no momento, uma vez que traria fim à dor dos sujeitos envolvidos. Tal compreensão leva o profissional a ter melhor aceitação da morte, pois a manutenção da vida do paciente é mais bem avaliada quando mantida com dignidade (LIMA AMM, et al, 2019).

Os significados que as pessoas em geral atribuem à morte de alguém depende de vivências, valores pessoais (MOTA MS, et al., 2011) e crenças religiosas (BORGES MS e MENDES N, 2012), fazendo-se presentes no resultado deste estudo. Esses significados, ao trazerem consigo a inevitabilidade da morte, minimizam as dificuldades sentidas (LIMA AMM, et al., 2019). Significar à morte dos pacientes baseados em aspectos como um "plano que foi finalizado", "missão cumprida" e "fim de uma etapa", corrobora com outras pesquisas realizadas (MARTINS LA, et al., 2019).

Acerca das possibilidades de intervenção, o suporte psicológico é reconhecido como necessário (MARTINS LA, et al., 2019). A educação para a morte foi percebida como importante, porém reconhece-se a dificuldade por parte dos profissionais, como da população em geral, de abordar tal assunto (PERBONI JS, et al., 2018), que na própria formação é trazido de forma superficial, conforme estudo realizado (FREITAS FRNN, et al., 2018).

Segundo Kovacs MJ (2016) educação para a morte vai além de aquisição de conhecimentos técnicos, envolvem valores e afetos. A autora traz ainda que não se trata de entregar receitas prontas, mas permitir questionamentos, como também desenvolvimento de empatia e sensibilidade em sujeitos de quaisquer idades.

Ademais, uma compreensão adequada sobre a de morte como parte da vida e sensação de o profissional colaborar com uma morte digna do paciente, estando este agindo ativamente nesse processo para uma boa morte, ameniza as dificuldades na convivência cotidiana com a morte (SANTOS QN, et al., 2020).

Nas UTIs em questão, todavia, não havia normativas que padronizassem a prática de cuidados paliativos, fato que pode colaborar com os sentimentos como impotência, por saber que existem possibilidades de atuação que não eram executadas. Conforme Barbosa AMGC e Massaroti L (2016), sentimentos de angústia surgem ao pensar no prolongamento do sofrimento do paciente com tratamentos vistos como desnecessários.

Perboni JS, et al. (2018) trazem que conhecimento sobre cuidados paliativos pode modificar as percepções do profissional frente à morte do paciente. Machado RS (2016) diz ainda que essa formação vem para compensar aquilo que estava em falta nas formações e que colaborava com a manutenção de sentimento de fracasso frente à morte de um paciente. Assim, tais conhecimentos possibilitam recursos para que esse momento seja passado com dignidade pelo paciente colaborando com seu bem-estar e conforto não apenas dele, mas também da equipe (SILVA RS, et al. 2015).

\section{CONCLUSÃO}

Foram constatados diferentes sentimentos e significados dos profissionais diante da morte e do morrer dos pacientes, que sofrem influência de fatores característicos do paciente, do profissional e da relação entre ambos. Enquanto profissionais de UTI, compreendem que precisam relacionar-se com a morte havendo, porém, maior sofrimento quando notam uma atuação distante da qualidade avaliada como necessária nessas circunstâncias. Independente da categoria, isso faz com que a prática vá de encontro com o objetivo de sua atuação profissional: o ato de cuidar. Desse modo, observa-se a importância de ações voltadas à educação para a morte considerando toda sua abrangência - técnica e relacional - para proporcionar a eles uma prática revestida de sentido. Por fim, apesar de existirem diversas pesquisas sobre a temática, observa-se uma escassez no que diz respeito a investigar as reações da equipe multiprofissional frente à morte de pacientes críticos, principalmente na região estudada. 


\section{REFERÊNCIAS}

1. ABREU RMD, et al. Motivos atribuídos por profissionais de uma Unidade de Terapia Intensiva para ausência no trabalho. Revista Brasileira de Enfermagem, 2014; 67(3); 386-393.

2. ALBUQUERQUE SGE, et al. Fatores de risco à segurança do enfermeiro na Unidade de Terapia Intensiva de um Hospital Geral. Revista Brasileira de Ciências da Saúde, 2015; 19(2): 135-142.

3. ARIES P. História da morte no Ocidente: da idade média aos nossos dias. Tradução Priscila Viana da Siqueira (Edição Especial). Rio de Janeiro: Nova Fronteira, 2017; 295p.

4. BARBOSA AMGC e MASSARONI L. Convivendo com a morte e o morrer. Rer. Enferm. UFPE on line, 2016, 10(2): 457-463.

5. BASTOS RA, et al. Angústias psicológicas vivenciadas por enfermeiros no trabalho com pacientes em processo de morte: um estudo clínico-qualitativo. Temas em Psicologia, 2018; 26(2), 795-805.

6. BORGES, MS e MARTINS N. Representaçoes de profissionais de saúde sobre a morte e o processo de morrer. Revista Brasileira de Enfermagem, 2012; 65(2); 324-331.

7. CARAM CS. Percepção dos profissionais acerca da morte de pacientes no contexto da Unidade de Terapia Intensiva. Revista de ciências da saúde nova esperança, 2018; 16(2): 48-57.

8. FREITAS, FRNN, et al. A morte e o morrer: representaçoes de graduandos em medicina. Revista Eletrônica Acervo Saúde, 2018; 11(1); 1-9.

9. GONÇALVES JR e SIMOES JRS. A percepçao do enfermeiro no lidar com a morte durante a assistência. Revista JRG de Estudos Acadêmicos, 2019; 2(5); 166-182.

10. HAYASIDA NMA, et al. Morte e luto: competências profissionais. Revista Brasileira de Terapias Cognitivas, 2014; 10(2): 112-121.

11. KOVACS MJ. Sofrimento da equipe de saúde no contexto hospitalar: cuidando do cuidador profissional, São Paulo. O mundo da saúde, 2010; 34(24): 420-429.

12. KOVACS MJ. Curso Psicologia da Morte: educação para a morte, São Paulo. Bol. Acad. Paulista de Psicologia, 2016; 36(91): 400-417.

13. LIMA AMM. Estratégias de enfrentamento pela equipe de enfermagem frente ao processo de morte e morrer em Unidades de Terapia Intensiva: uma revisão integrativa. Arquivos Brasileiros de Medicina Naval, 2019; 80(1): 57-65.

14. MACHADO RS. Finitude e morte na sociedade ocidental: uma reflexão com foco nos profissionais de saúde, Valência. Cultura Cuidado, 2016; 20(45): 91-97.

15. MARTINS LA, et al. Significado da morte de pacientes para profissionais de saúde em unidade de terapia intensiva. REFACS (online), 2019; 7(4): 448-457.

16. MONTEIRO JK. Sofrimento psíquico de trabalhadores de Unidade de Terapia Intensiva. Revista Psicologia: Organizaçoes e Trabalho, 2012; 12(2): 245-250.

17. MOTA MS, et al. Reaçoes e sentimentos de profissionais da enfermagem frente à morte dos pacientes sob seus cuidados. Rev. Gaúcha Enferm., 2011; 32(1); 129-135.

18. NAVAIS MC, et al. Morte e luto: vivências de pediatras em unidades de terapia intensiva pediátra e neonatal nas microrregioes de Barbacena e Sao Joao Del Rei. Rev. Med. Minas Gerais, 2017; 27(1); 60-65.

19. PERBONI JS, et al. Profissionais de saúde e o processo de morte e morrer dos pacientes: uma revisão integrativa. Persona y Bioética, 2018; 22(2): 288-302.

20. PEREIRA NETO HS, et al. Promovendo instituições eficazes, cenas da unidade de terapia intensiva: a morte e o morrer, sob o olhar do profissional de enfermagem. Rev. Psicologia e Saúde em Debate, 2019; 5(1); 1-9.

21. SANTOS QN, et al. Significados de morte e morrer para profissionais de unidade de terapia intensiva. Psicologia Argumento, 2020; 38(100): 316-337.

22. SCARTON J, et al. Enfermagem: a morte e o morrer em unidade de terapia intensiva pediátrica e neonatal. Rev. Enferm. UFPE on line, 2013; 7(10): 5929-5937.

23. SILVA RS, et al. Conforto para uma boa morte: perspectiva de uma equipe de enfermagem intensiva. Esc. Anna Nery, 2015; 19(1): 40-46.

24. SOUZA PSN e CONCEIÇAO AOF. Processo de morrer em unidade de terapia pediátrica. Rev. Bioét., 2018; v26(1); 127-134.

25. VIERAS AR e PIO DAM. Morte na UTI pediátrica (UTI): experiências e percepçoes de profissionais. RIES, 2018; 7(1), 454-466.

26. VICENSI, MC. Reflexão sobre a morte e o morrer na UTI: a perspectiva do profissional. Rev. Bioét., 2016; 24(1); 6472. 